$\mathrm{PFC} / \mathrm{JA}-89-43$

SELF-FIELD-INDUCED CHAOTIC ELECTRON MOTION

IN FREE ELECTRON LASERS

by

Chiping Chen and Ronald C. Davidson

September, 1989

Plasma Fusion Center

Massachusetts Institute of Technology

Cambridge, MA 02139

Presented at the Eleventh International IEEE Conference on Free Electron Lasers, Naples, Florida.

Research supported by the Department of Energy High Energy Physics Division, the Office of Naval Research, and the Naval Research Laboratory Plasma Physics Division. 


\title{
Self-Field-Induced Chaotic Electron Motion In Free Electron Lasers
}

\author{
Chiping Chen and Ronald C. Davidson \\ Plasma Fusion Center \\ Massachusetts Institute of Technology \\ Cambridge, Massachusetts 02139, U.S.A.
}

\begin{abstract}
It is shown that the inclusion of the equilibrium self fields of the electron beam results in chaotic electron motion in the field configuration consisting of a constant-amplitude helical wiggler magnetic field and a uniform axial guide field. The threshold value of the self-field parameter $\epsilon=\omega_{p b}^{2} / 4 \Omega_{c}^{2}$ is determined for the onset of chaoticity in the Group-I and Group-II orbits for the parameter regime corresponding to moderately high beam current (and density). The time scale for self-field-induced changes in the particle orbits is found to be of the order of beam-transit time through one wiggler period.
\end{abstract}

\section{INTRODUCTION}

The free electron laser (FEL) makes use of the unstable interaction of a relativistic electron beam with a transverse wiggler magnetic field to generate coherent electromagnetic radiation [1]. In recent experiments [2-5], megawatts to gigawatts of coherent radiation have been generated in the submillimeter to millimeter wavelength range. Several FEL experiments [3-5] operate at moderately high current and make use of a magnetic guide field $B_{0} \vec{e}_{z}$ to steer the electron beam in the axial direction. If the equilibrium self-electric and self-magnetic fields [6] produced by the nonneutral electron beam are negligible, the helical wiggler magnetic field $\vec{B}_{w}(z)=-B_{w}\left(\vec{e}_{x} \cos k_{w} z+\vec{e}_{y} \sin k_{w} z\right)$ and the axial guide field $B_{0} \vec{e}_{z}$ then act in combination to affect the particle motion and determine the detailed properties of the FEL interaction [7-9]. For high-current FEL operation, however, the equilibrium self-electric and self-magnetic fields can significantly alter the electron orbits relative to those in the vacuum field, which is the subject matter of this paper. In addition, the wiggler-induced electron current generates a magnetostatic 
field which changes the effective amplitude of the wiggler field [10].

It is shown in this paper that the inclusion of the equilibrium radial self-electric and azimuthal self-magnetic fields destroys the integrability of the motion of a relativistic electron in the field configuration consisting of a constant-amplitude helical wiggler magnetic field $\vec{B}_{w}(z)$ and a uniform axial magnetic field $B_{0} \vec{e}_{z}$, and consequently part of the phase space is chaotic. As an example, for beam radius $r_{b}=0.31 \mathrm{~cm}$, axial guide field $B_{0}=14.2 \mathrm{kG}$, wiggler amplitude $B_{w}=710 \mathrm{G}$, wiggler wavelength $\lambda_{w}=2 \pi / k_{w}=3.0 \mathrm{~cm}$, and relativistic mass factor $\gamma_{b}=3.0$, it is shown in Sec. IV that equilibrium self-field effects cause fully developed chaoticity in the electron orbits whenever the beam current $I_{b}$ exceeds the threshold beam current $I_{b}^{\text {th }}=4.3 \mathrm{kA}$.

A Hamiltonian system with $N$ degrees of freedom is integrable if it has $N$ independent constants of motion in involution, i.e., the Poisson bracket of any pair of them vanishes. If the number of constants is less than $N$, then the motion is nonintegrable and part of phase space is chaotic in the sense that adjacent initial conditions lead to exponentially divergent trajectories. Typically, however, there are also regular regions in phase space, consisting of the Kolmogorov-Arnold-Moser (KAM) surfaces that limit the chaotic regions of phase space [11]. The Poincaré surface-of-section method is useful in analyzing nonintegrable systems.

\section{MODEL AND ASSUMPTIONS}

Consider the motion of a relativistic electron in the field configuration consisting of a uniform axial magnetic field $B_{0} \vec{e}_{z}$, a constant-amplitude helical wiggler magnetic field $\vec{B}_{w}=-B_{w}\left(\vec{e}_{x} \cos k_{w} z+\vec{e}_{y} \sin k_{w} z\right)$, and the radial self-electric and azimuthal selfmagnetic fields produced by a relativistic nonneutral electron beam with radius $r_{b}$, average axial velocity $V_{b} \vec{e}_{z}$, and uniform density profile [12]

$$
n_{b}^{0}(r)= \begin{cases}n_{b}=\text { const. }, & 0 \leq r<r_{b} \\ 0, & r>r_{b}\end{cases}
$$

where $r=\left(x^{2}+y^{2}\right)^{1 / 2}$ is the radial distance from the beam center. The equations of 
motion for an electron within the beam $\left(0 \leq r<r_{b}\right)$ can be derived from the Hamiltonian

$$
H=\left[(c \vec{P}+e \vec{A})^{2}+m^{2} c^{4}\right]^{1 / 2}-e \Phi_{s} \equiv \gamma m c^{2}-e \Phi_{s}
$$

where $\gamma=\left[1+(\vec{p} / m c)^{2}\right]^{1 / 2}$ is the relativistic mass factor, and the scalar and vector potentials, $\Phi_{s}$ and $\vec{A}$, are defined by

$$
\begin{gathered}
\Phi_{s}=\frac{m \omega_{p b}^{2}}{4 e}\left(x^{2}+y^{2}\right) \\
\vec{A}=B_{0} x \vec{e}_{y}+A_{w}\left(\vec{e}_{x} \cos k_{w} z+\vec{e}_{y} \sin k_{w} z\right)+\beta_{b} \Phi_{s} \vec{e}_{z}
\end{gathered}
$$

Here, $A_{w}=B_{w} / k_{w}=$ const, $\beta_{b}=V_{b} / c$ is the normalized beam velocity, $-\mathrm{e}$ and $\mathrm{m}$ are the electron charge and rest mass, respectively, $\mathrm{c}$ is the speed of light in vacuo, and $\omega_{p b}=\left(4 \pi e^{2} n_{b} / m\right)^{1 / 2}$ is the nonrelativistic plasma frequency of the beam electrons. The mechanical momentum $\vec{p}$ is related to the canonical momentum $\vec{P}$ by $\vec{p}=\vec{P}+e \vec{A} / c$. Because $H$ is independent of time, the total energy of an individual electron, $\gamma m c^{2}-e \Phi_{s}$, is a constant of the motion.

In order to find an additional constant of the motion and determine the resonances, we have performed the canonical transformation to the new variables $\varphi, \psi, z^{\prime}, P_{\varphi}, P_{\psi}, P_{z^{\prime}}$ [12], i.e.,

$$
\begin{gathered}
x=\left(2 P_{\varphi} / m \Omega_{c}\right)^{1 / 2} \sin \left(\varphi+k_{w} z^{\prime}\right)-\left(2 P_{\psi} / m \Omega_{c}\right)^{1 / 2} \cos \left(\psi-k_{w} z^{\prime}\right) \\
y=\left(2 P_{\varphi} / m \Omega_{c}\right)^{1 / 2} \sin \left(\psi-k_{w} z^{\prime}\right)-\left(2 P_{\psi} / m \Omega_{c}\right)^{1 / 2} \cos \left(\varphi+k_{w} z^{\prime}\right) \\
z=z^{\prime} \\
P_{x}=\left(2 m \Omega_{c} P_{\varphi}\right)^{1 / 2} \cos \left(\varphi+k_{w} z^{\prime}\right) \\
P_{y}=\left(2 m \Omega_{c} P_{\psi}\right)^{1 / 2} \cos \left(\psi-k_{w} z^{\prime}\right) \\
P_{z}=P_{z^{\prime}}-k_{w} P_{\varphi}+k_{w} P_{\psi}
\end{gathered}
$$

where $\Omega_{c}=e B_{0} / m c$ is the nonrelativistic cyclotron frequency. It is shown in Sec. III that the canonical momenta $P_{\varphi}$ and $P_{\psi}$ characterize, respectively, the gyroradius and the 
guiding center radius of the steady-state orbits. The Hamiltonian in the new variables $\left(\varphi, \psi, z^{\prime}, P_{\varphi}, P_{\psi}, P_{z^{\prime}}\right)$ is given by

$$
\begin{gathered}
\frac{1}{m c^{2}} H\left(\varphi, \psi, P_{\varphi}, P_{\psi}, P_{z^{\prime}}\right)=-\frac{e \Phi s}{m c^{2}}+ \\
{\left[\frac{2 \Omega_{c} P_{\varphi}}{m c^{2}}+\frac{2 e A_{w}}{m c^{2}}\left(\frac{2 \Omega_{c} P_{\varphi}}{m c^{2}}\right)^{\frac{1}{2}} \cos \varphi+\left(\frac{P_{z^{\prime}}-k_{w} P_{\varphi}+k_{w} P_{\psi}}{m c}+\beta_{b} \frac{e \Phi_{s}}{m c^{2}}\right)^{2}+\left(\frac{e A_{w}}{m c^{2}}\right)^{2}+1\right]^{\frac{1}{2}}}
\end{gathered}
$$

where $\Phi_{s}$ and the dimensionless equilibrium self-field parameter $\epsilon$ are defined by

$$
\begin{gathered}
e \Phi_{s}=2 \epsilon \Omega_{c}\left[P_{\varphi}+P_{\psi}-2\left(P_{\varphi} P_{\psi}\right)^{1 / 2} \sin (\varphi+\psi)\right], \\
\epsilon=\frac{\omega_{p b}^{2}}{4 \Omega_{c}^{2}} .
\end{gathered}
$$

The self-field parameter $\epsilon$ characterizes the strength of the equilibrium self fields relative to the focusing force produced by the axial guide field $B_{0} \vec{e}_{z}$. By introducing the dimensionless parameters and variables, i.e.,

$$
\begin{gathered}
\hat{\Omega}_{c}=\Omega_{c} / c k_{w}, \hat{\omega}_{p b}=\omega_{p b} / c k_{w}, \hat{\Phi}_{s}=e \Phi s / m c^{2}, a_{w}=e A_{w} / m c^{2} \\
\hat{P}_{z}=P_{z} / m c, \hat{P}_{\varphi}=k_{w} P_{\varphi} / m c, \hat{P}_{\psi}=k_{w} P_{\psi} / m c, \hat{H}=H / m c^{2} \\
\tau=c k_{w} t, \hat{z}^{\prime}=k_{w} z^{\prime}
\end{gathered}
$$

the Hamiltonian defined in Eq. (6) can be expressed in the dimensionless form

$$
\begin{gathered}
\hat{H}\left(\varphi, \psi, \hat{P}_{\varphi}, \hat{P}_{\psi}, \hat{P}_{z^{\prime}}=\text { const. }\right)= \\
{\left[2 \hat{\Omega}_{c} \hat{P}_{\varphi}+2 a_{w}\left(2 \hat{\Omega}_{c} \hat{P}_{\varphi}\right)^{1 / 2} \cos \varphi+\left(\hat{P}_{z^{\prime}}-\hat{P}_{\varphi}+\hat{P}_{\psi}+\beta_{b} \hat{\Phi}_{s}\right)^{2}+a_{w}^{2}+1\right]^{1 / 2}-\hat{\Phi}_{s}=\text { const. }}
\end{gathered}
$$

where the normalized self-field potential is given by

$$
\hat{\Phi}_{s}\left(\varphi, \psi, \hat{P}_{\varphi}, \hat{P}_{\psi}\right)=2 \epsilon \hat{\Omega}_{c}\left[\hat{P}_{\varphi}+\hat{P}_{\psi}-2\left(\hat{P}_{\varphi} \hat{P}_{\psi}\right)^{1 / 2} \sin (\varphi+\psi)\right]
$$

Because $\hat{H}$ is independent of $\hat{z}^{\prime}$, it follows that $\hat{P}_{z^{\prime}}=$ const. For $\epsilon \neq 0$, there are apparently only two constants of the motion and chaotic behavior is expected. Note that the present analysis is restricted to a class of FELs with nonzero axial guide field $B_{0} \vec{e}_{z}$. [The canonical transformation in Eq. (5) becomes singular as $B_{0} \rightarrow 0$.] 


\section{INTEGRABLE LIMIT $(\epsilon=0)$}

In the $\epsilon=0$ limit $\left(\hat{\Phi}_{s}=0\right)$, the Hamiltonian $\hat{H}$ in Eq. (10) reduces to

$$
\begin{gathered}
\hat{H}_{0}\left(\varphi, \hat{P}_{\varphi}, \hat{P}_{\psi}, \hat{P}_{z^{\prime}}\right)= \\
{\left[2 \hat{\Omega}_{c} \hat{P}_{\varphi}+2 a_{w}\left(2 \hat{\Omega}_{c} \hat{P}_{\varphi}\right)^{1 / 2} \cos \varphi+\left(\hat{P}_{z^{\prime}}-\hat{P}_{\varphi}+\hat{P}_{\psi}\right)^{2}+a_{w}^{2}+1\right]^{1 / 2}=\gamma_{0}}
\end{gathered}
$$

which possesses the constants of the motion $\hat{P}_{\psi}, \hat{P}_{z^{\prime}}$ and $\gamma_{0}$. The motion is integrable and has been analyzed by several authors [7-9]. The steady-state orbits are given by

$$
\begin{gathered}
\cos \varphi_{0}= \pm 1 \\
\left(2 \hat{\Omega}_{c} \hat{P}_{\varphi 0}\right)^{1 / 2}= \pm \frac{a_{w} \hat{\Omega}_{c}}{\hat{p}_{z 0}-\hat{\Omega}_{c}}>0
\end{gathered}
$$

where $\hat{p}_{z 0}=\hat{P}_{z^{\prime}}-\hat{P}_{\varphi 0}+\hat{P}_{\psi}$ is the normalized axial mechanical momentum. To locate the steady-state orbits for given electron energy $\gamma_{0}, \hat{p}_{z 0}$ has to be solved from the equation

$$
\hat{p}_{z 0}^{2}\left[1+\frac{a_{w}^{2}}{\left(\hat{p}_{z 0}-\hat{\Omega}_{c}\right)^{2}}\right]+1=\gamma_{0}^{2} .
$$

Equation (14) has at most four real roots, including the Group-I stable orbit $\left(\hat{p}_{z 0}>\hat{\Omega}_{c}\right)$ that exists for $0<\hat{\Omega}_{c}<\hat{\Omega}_{c}^{c r}$ and merges with an unstable orbit at $\hat{\Omega}_{c}=\hat{\Omega}_{c}^{c r}$ and the Group-II stable orbit $\left(0<\hat{p}_{z 0}<\hat{\Omega}_{c}\right)$ that exists for all $\hat{\Omega}_{c}>0$. In FEL operation, the electron beam is injected into the Group-I or Group-II orbit. Typical phase-space structure is plotted in Fig. 1 for the two cases $0<\hat{\Omega}_{c}<\hat{\Omega}_{c}^{c r}$ and $\hat{\Omega}_{c}>\hat{\Omega}_{c}^{c r}$, where the elliptic (hyperbolic) fixed points correspond to the stable (unstable) steady-state orbits. The Group-I orbit has greater axial momentum than the Group-II orbit in Fig. 1(a), whereas only the Group-II orbit is present in Fig. 1(b). The frequency of the betatron oscillations about the Group-I or Group-II orbit is given by [12]

$$
\hat{\omega}=\frac{\left|\hat{p}_{z 0}-\hat{\Omega}_{c}\right|}{\gamma_{0}}\left[1-\frac{\hat{\Omega}_{c}}{a_{w}}\left(\frac{\hat{p}_{t 0}}{\hat{p}_{z 0}}\right)^{3}\right]^{\frac{1}{2}}
$$

where $\hat{p}_{t 0}=a_{w} \hat{p}_{z 0} /\left(\hat{p}_{z 0}-\hat{\Omega}_{c}\right)$ is the normalized transverse mechanical momentum. For $\left|\hat{p}_{t 0} / \hat{p}_{z 0}\right|^{3}<<a_{w} / \hat{\Omega}_{c}$, it follows from Eq. (15) that $\hat{\omega} \cong\left|\hat{p}_{z 0}-\hat{\Omega}_{c}\right| / \gamma_{0}$, thereby recovering 
the results in Ref. [7]. In addition, the steady-state trajectories can be expressed in dimensional variables as [12]

$$
\begin{gathered}
x(t)= \pm\left(2 P_{\varphi 0} / m \Omega_{c}\right)^{1 / 2} \sin \left[k_{w} z(t)\right]-\left(2 P_{\psi 0} / m \Omega_{c}\right)^{1 / 2} \cos \psi_{0} \\
y(t)=\mp\left(2 P_{\varphi 0} / m \Omega_{c}\right)^{1 / 2} \cos \left[k_{w} z(t)\right]+\left(2 P_{\psi 0} / m \Omega_{c}\right)^{1 / 2} \sin \psi_{0} \\
z=z_{0}+v_{z 0} t
\end{gathered}
$$

for $\cos \varphi_{0}= \pm 1$. Equation (16) describes helical trajectories with gyroradius $r_{c}=$ $\left(2 P_{\varphi 0} / m \Omega_{c}\right)^{1 / 2}$ and guiding center radius $r_{g}=\left(2 P_{\psi_{0}} / m \Omega_{c}\right)^{1 / 2}$.

\section{CHAOTIC MOTION $(\epsilon \neq 0)$}

The equilibrium self-field contribution $\hat{\Phi}_{\text {s }}$ in Eq. (10) invalidates the constancy of $\hat{P}_{\psi}$. The motion given by the Hamiltonian $\hat{H}$ in Eq. (10) occurs in the three-dimensional phase space $\left(\varphi, \psi, \hat{P}_{\varphi}\right)$, because $\hat{P}_{\psi}$ is solved from $\hat{H}=$ const. The time scale $T_{s}$ for the self-field-induced changes in the particle orbit can be estimated from the rate of change of the phase $\psi$ in the self-field potential $\hat{\Phi}_{s}$ defined in Eq. (11). It follows from $d \psi / d \tau=\partial \hat{H} / \partial \hat{P}_{\psi}=\hat{p}_{z} / \gamma+O(\epsilon) \cong \beta_{b}$ or $d \psi / d t \cong k_{w} V_{b}$ that the time required for $\psi$ to advance by $2 \pi$ is given by

$$
T_{s}=\frac{\lambda_{w}}{V_{b}}
$$

where $\lambda_{w}=2 \pi / k_{w}$ is the wiggler period, and $\hat{p}_{z}=\hat{P}_{z^{\prime}}-\hat{P}_{\varphi}+\hat{P}_{\psi}+\beta_{b} \hat{\Phi}_{s}\left(\varphi, \psi, \hat{P}_{\varphi}, \hat{P}_{\psi}\right)$ is the normalized axial mechanical momentum.

For $\epsilon<<1$, in the vicinity of the Group-I or Group-II orbit, the particle motion occurs on a torus as illustrated in Fig. 2. In Fig. 2, the toroidal and poloidal angles designate, respectively, $\psi$ and the phase of $\delta \varphi=\varphi-\varphi_{0}\left(\cos \varphi_{0}= \pm 1\right)$ or $\delta \hat{P}_{\varphi}=\hat{P}_{\varphi}-\hat{P}_{\varphi 0}$, the dashed-line toroidal circle represents the Group-I or Group-II orbit, and $\beta_{b}$ and $\hat{\omega}$ are the normalized rotation rates of the two angles. Clearly, an approximate resonance condition is

$$
n \hat{\omega}+m \beta_{b}=0
$$


where $\mathrm{m}$ and $\mathrm{n}$ are integer, and the betatron oscillation frequency $\hat{\omega}$ is defined in Eq. (15). A detailed resonance analysis has been carried out in Ref. [12]. For $m=1$, the resonance condition and the resonance width $\hat{w}_{n}$ are, respectively, given by

$$
\begin{gathered}
n \frac{\left|\hat{p}_{z 0}-\hat{\Omega}_{c}\right|}{\gamma_{0}}\left[1-\frac{\hat{\Omega}_{c}}{a_{w}}\left(\frac{\hat{p}_{t 0}}{\hat{p}_{z 0}}\right)^{3}\right]^{\frac{1}{2}}+\frac{\hat{p}_{z 0}}{\gamma_{0}}-2 \epsilon \hat{\Omega}_{c}\left(1-\beta_{b} \frac{\hat{p}_{z 0}}{\gamma_{0}}\right)=0, \\
\hat{w}_{n}=\left[32 \epsilon \gamma_{b} \hat{\Omega}_{c}\left|J_{n}\left(\delta \varphi_{0}\right)\right|\right]^{1 / 2}\left(\hat{P}_{\varphi 0} \hat{P}_{\psi 0}\right)^{1 / 4}=4\left[\frac{\gamma_{b} r_{c} r_{g} I_{b}\left|J_{n}\left(\delta \varphi_{0}\right)\right|}{\beta_{b} r_{b}^{2} I_{A}}\right]^{\frac{1}{2}} .
\end{gathered}
$$

Here, $r_{b}, I_{b}$ and $\gamma_{b} m c^{2}$ are the radius, current and energy of the electron beam, $I_{A}=$ $m c^{3} / e \cong 17 \mathrm{kA}$ is the Alfvén current, and $r_{c}$ and $r_{g}$ are the gyroradius and the guiding center radius, respectively. Figure 3 shows plots of the resonance curves (the solid curves) corresponding to the solutions to Eq. (19) for $\epsilon=0.01$ and $n=-1,-2$ and -3 . The dashed curves in Fig. 3 are the integrable steady-state orbits calculated from Eq. (14). When the resonance curves of order $\mathrm{n}$ intersect the integrable stable steady-state orbits, islands of order $n$ are expected to appear in the phase space. Also, the threshold values of the self-field parameter $\epsilon$ can be estimated for the onset of chaoticity, using the scaling relation in Eq. (20) and the resonance-overlap criterion [11].

In order to demonstrate that the particle motion is indeed chaotic, Poincare surfaceof-section maps have been generated by numerically integrating the equations of motion derived from the Hamiltonian in Eq. (10). Figure 4 shows typical nonintegrable surfaceof-section plots for $\epsilon=0.01$ and for the two cases: (a) $0<\hat{\Omega}_{c}<\hat{\Omega}_{c}^{c r}$, and (b) $\hat{\Omega}_{c}>\hat{\Omega}_{c}^{c r}$. The integrable limits corresponding to Figs. $4(\mathrm{a})$ and $4(\mathrm{~b})$ are shown in Figs. 1(a) and $1(\mathrm{~b})$, respectively. In Fig. 4 , the initial condition for $\hat{P}_{\psi}$ is fixed at the value $\left(2 \hat{P}_{\psi 0} / \hat{\Omega}_{c}\right)^{1 / 2}=0.25$, whereas the initial condition for $\hat{p}_{z}$ is allowed to vary. The secondorder island appearing near the Group-II orbit in Fig. 4(b) manifests the intersection between the $n=-2$ resonance curve and the Group-II orbit at $\hat{\Omega}_{c}=4.0$ in Fig. 3 . It is evident that the self fields $(\epsilon=0.01)$ are not strong enough to cause high-degree chaoticity in the vicinity of either the Group-I orbit in Fig. 4(a) or the Group-II orbit in Fig. 4(b). The onset of chaos for the Group-II orbit [corresponding to Fig. 4(b)] occurs at $\epsilon=0.04$ and is shown in Fig. 5, where $\hat{\Omega}_{c}=4.0, \hat{H}=3.0, a_{w}=0.2, \beta_{b}=0.93$ 
and $k_{w} r_{b}=0.65$. As an example, for $\lambda_{w}=3.0 \mathrm{~cm}$, the dimensionless parameters in Fig. 6 correspond to $r_{b}=0.31 \mathrm{~cm}, I_{b}=4.3 \mathrm{kA}, B_{w}=710 \mathrm{G}, B_{0}=14.2 \mathrm{kG}, \beta_{b}=0.93$ and $\gamma_{b}=3.0$.

It should be pointed out that in contrast to the chaoticity induced by an electromagnetic perturbation with time scale characterized by the synchrotron period of an electron moving in the ponderomotive potential [13], chaotic behavior arising from equilibrium self-field effects is likely to be more harmful to FEL operation. It is known that sidebands also cause chaotic behavior $[14,15]$, which is not likely to be important until nonlinear saturation occurs.

\section{CONCLUSIONS}

We have investigated the effects of equilibrium self fields on the electron orbits in a constant-amplitude helical-wiggler FEL with an axial guide field $B_{0} \vec{e}_{z}$. It was shown that the equilibrium self fields destroy the integrability of the motion and consequently part of phase space becomes chaotic. The threshold values of the self-field parameter $\epsilon=\omega_{p b}^{2} / 4 \Omega_{c}$ were determined for the onset of chaos in the parameter regime corresponding to multi-kiloampere beam current and moderately relativistic electron beam. Further investigations are required to understand how self-field-induced chaoticity alters the stability properties of the FEL interaction.

\section{ACKNOWLEDGEMENTS}

This work was supported by the Department of Energy High Energy Physics Division, the Office of Naval Research, and the Naval Research Laboratory Plasma Physics Division. 


\section{REFERENCES}

1. T.C. Marshall, Free Electron Lasers (Macmillan, New York, 1985).

2. T. J. Orzechowski, et al, Phys. Rev. Lett. 57, 2172 (1986).

3. J. Masud, et al, Phys. Rev. Lett. 56, 1567 (1986).

4. J. Fajans, G. Bekef, Y.Z. Yin and B. Lax, Phys. Fluids 28, 1995 (1985).

5. R.K. Parker, et al, Phys. Rev. Lett. 48, 238 (1982).

6. R.C. Davidson, Theory of Nonneutral Plasmas, (Addison-Wesley, Reading, Massachusetts, 1989).

7. H.P. Freund, Phys. Rev. A27, 1977 (1983); H.P. Freund and A. K. Ganguly, Phys. Rev. A28, 3438 (1983); H.P. Freund, et al, Phys. Rev. A26, 2004 (1982); H.P.

Freund and A.T. Drobot, Phys. Fluids 25, 736 (1982).

8. R.C. Davidson and H.S. Uhm, J. Appl. Phys. 53, 2910 (1982).

9. L. Friedland, Phys. Fluids 23, 2376 (1980).

10. N.S. Ginzburg, IEEE Trans. Plasma Sci. PS-15, 411 (1987).

11. A.J. Lichtenberg and M.A. Lieberman, Regular and Stochastic Motion (SpringerVerlag, New York, 1983).

12. C. Chen and R.C. Davidson, submitted to Phys. Fluids B (1989).

13. C. Chen and G. Schmidt, Comments in Plasma Physics and Controlled Fusion 12, $83(1988)$.

14. R.C. Davidson and J.S. Wurtele, Phys. Fluids 30, 557 (1987).

15. S. Riyopoulos and C.M. Tang, Nucl. Instrum. Methods A259, 226 (1987). 


\section{FIGURE CAPTIONS}

Fig. 1. Contour plots in the integrable phase plane $\left(\varphi, \hat{p}_{z}\right)$ calculated from Eq. (12) for $\epsilon=0, \gamma_{0}=3.0$ and $a_{w}=0.2$. The two cases correspond to (a) $\hat{\Omega}_{c}=2.0$ $<\hat{\Omega}_{c}^{c r} \cong 2.1$, and $(b) \hat{\Omega}_{c}=4.0>\hat{\Omega}_{c}^{c r}$.

Fig. 2. The torus structure in the vicinity of the stable steady-state orbit $\left(\varphi_{0}, \hat{P}_{\varphi 0}\right)$.

Fig. 3. The equilibrium self-field resonance curves (solid curves) correspond to the solutions to Eq. (19) for $n=-1,-2,-3, \epsilon=0.01, \gamma_{0}=3.0$ and $a_{w}=0.2$. The dashed curves are the integrable steady-state orbits calculated from Eq. (14).

Fig. 4. Typical nonintegrable surface-of-section plots with $\psi=0$, $\bmod 2 \pi$, for the two cases (a) $0<\hat{\Omega}_{c}=2.0<\hat{\Omega}_{c}^{c r} \cong 2.1$, and (b) $\hat{\Omega}_{c}=4.0>\hat{\Omega}_{c}^{c r}$. Other system parameters are (a) $\epsilon=0.01, \hat{H} \doteq 3.0, a_{w}=0.2$ and $\beta_{b}=0.91$, and (b) $\epsilon=0.01$, $\hat{H}=3.0, a_{w}=0.2$ and $\beta_{b}=0.93$.

Fig. 5. The surface-of-section plot at the onset of chaoticity of the Group-II orbit for the choice of system parameters $\epsilon=0.04, \hat{\Omega}_{c}=4.0, \hat{H}=3.0, a_{w}=0.2$ and $\beta_{b}=0.93$. In this plot, the normalized effective gyroradius $\left(2 \hat{P}_{\varphi} / \hat{\Omega}_{c}\right)^{1 / 2}$ ranges from 0.17 to 0.35 , the normalized guiding center radius is $\left(2 \hat{P}_{\psi} / \hat{\Omega}_{c}\right)^{1 / 2} \cong 0.25$, and the normalized beam radius is $k_{w} r_{b}=0.65$. 


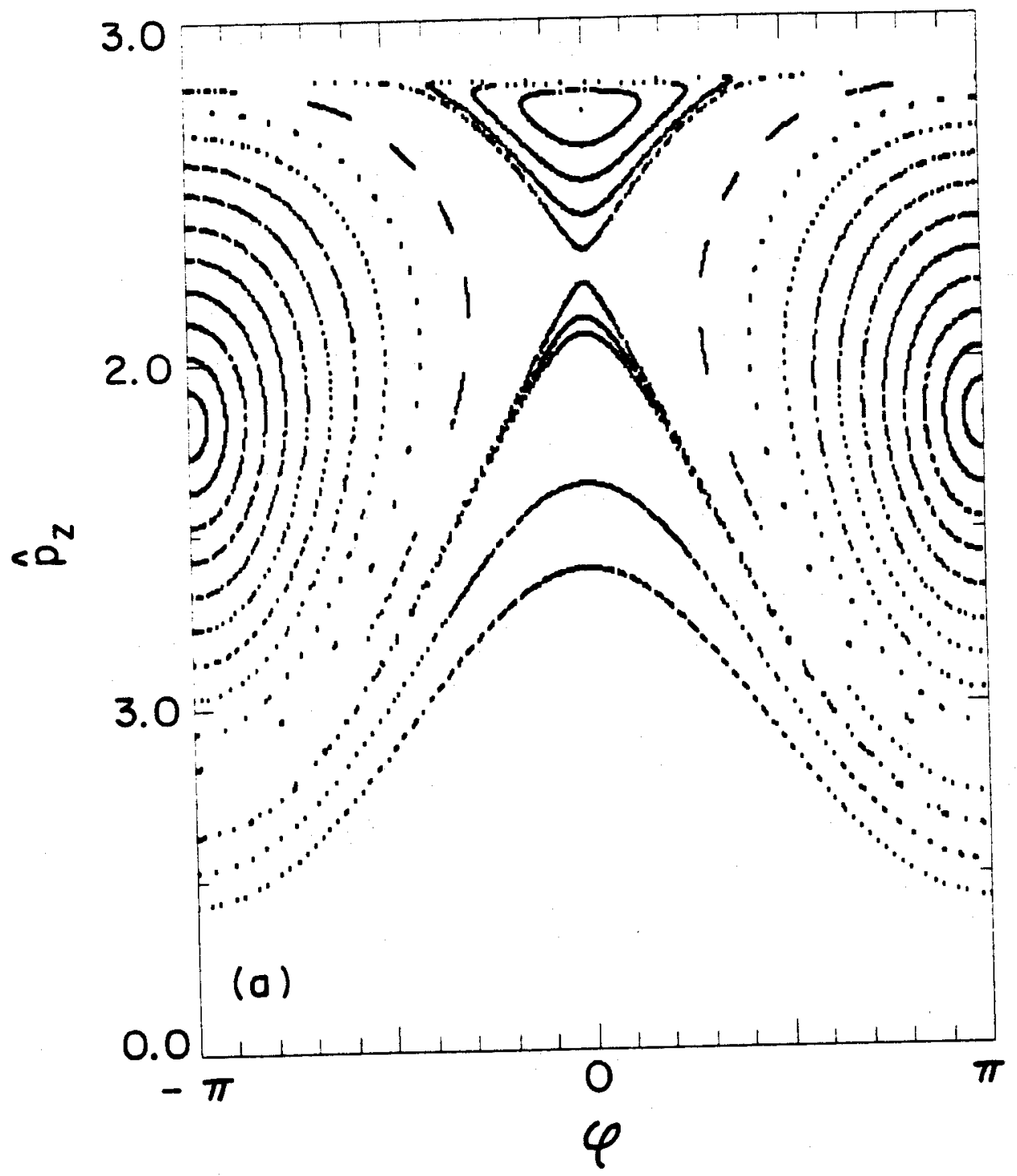

Figure la 


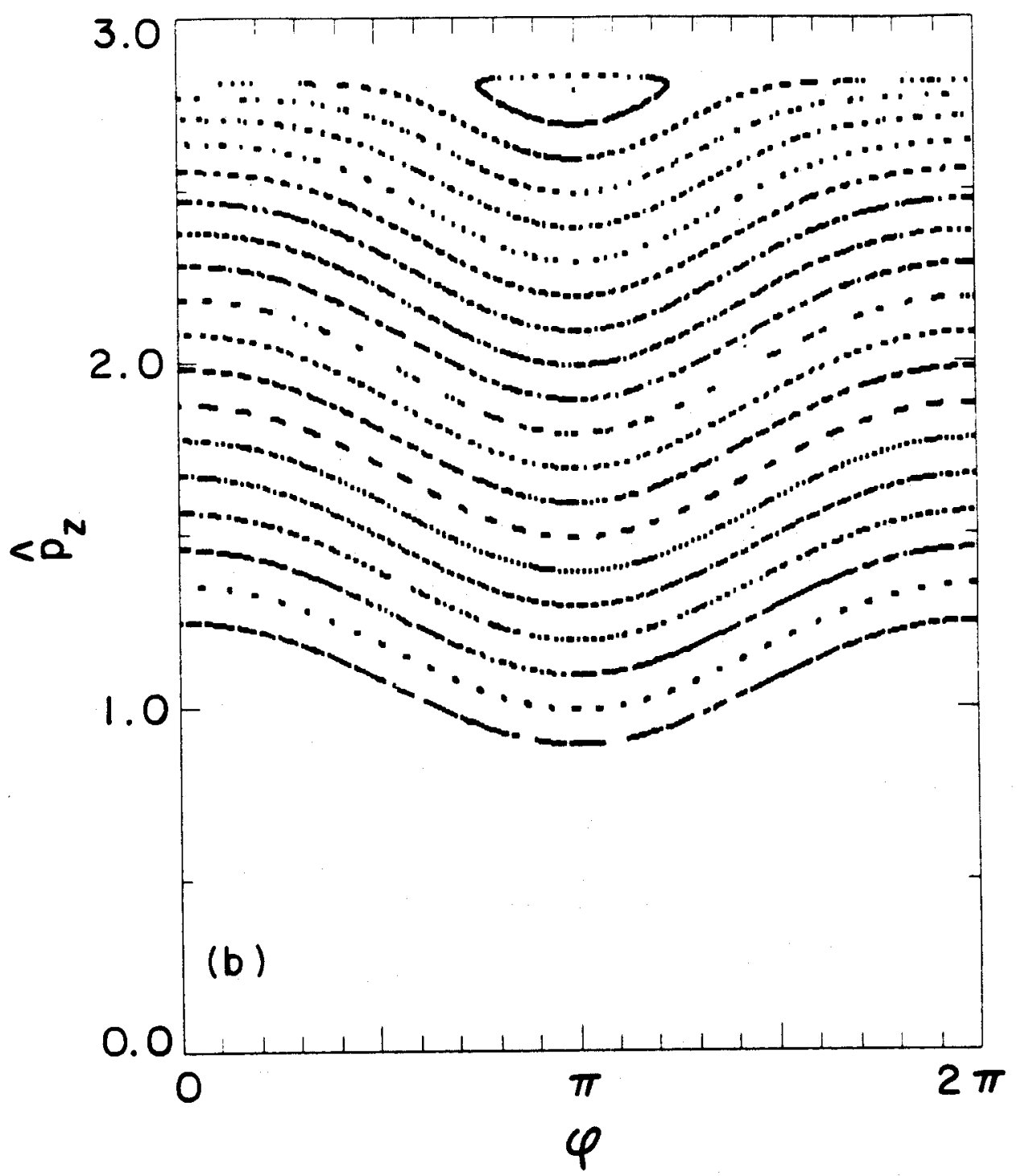

Figure 1b 


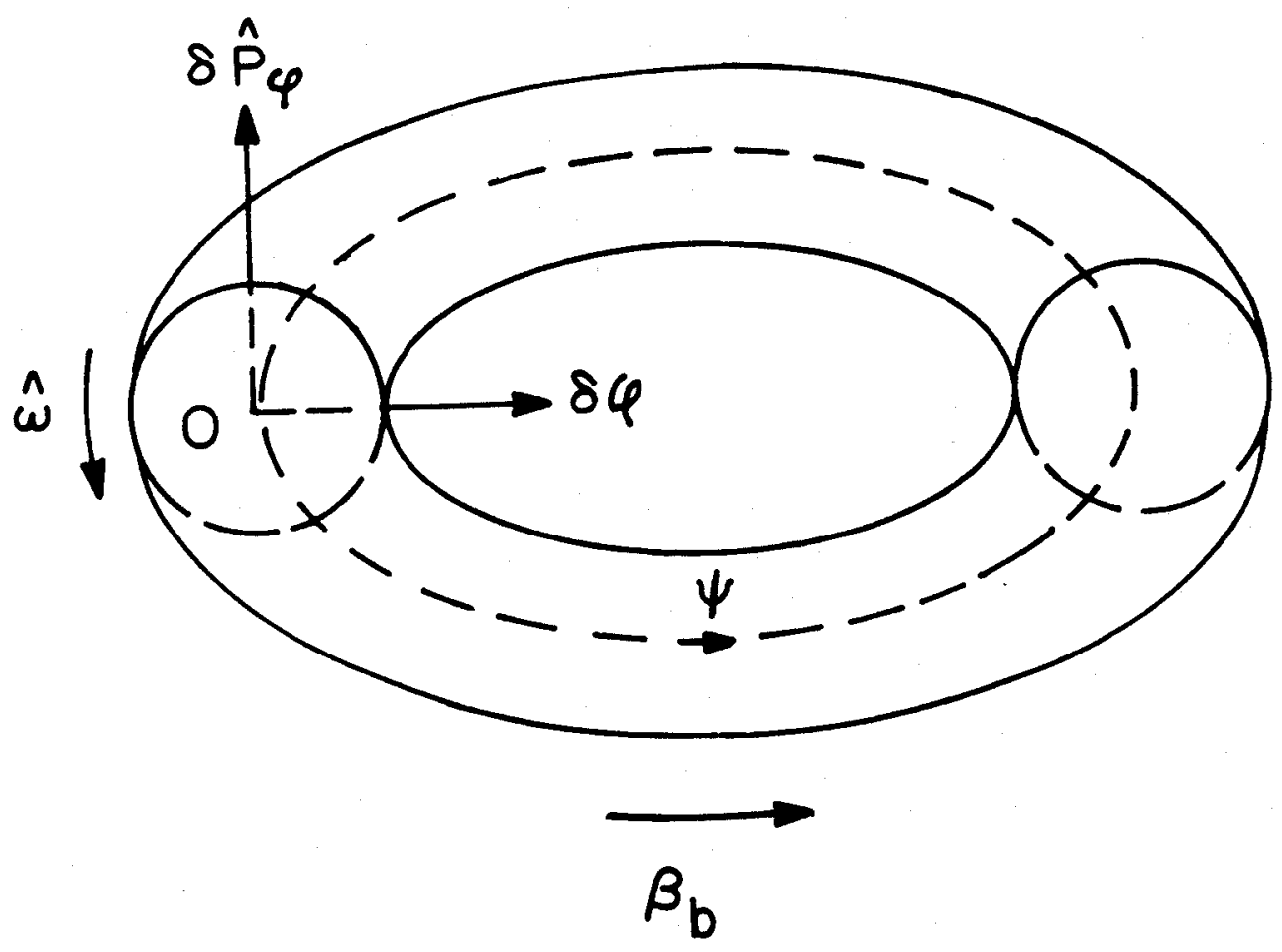

Figure 2 


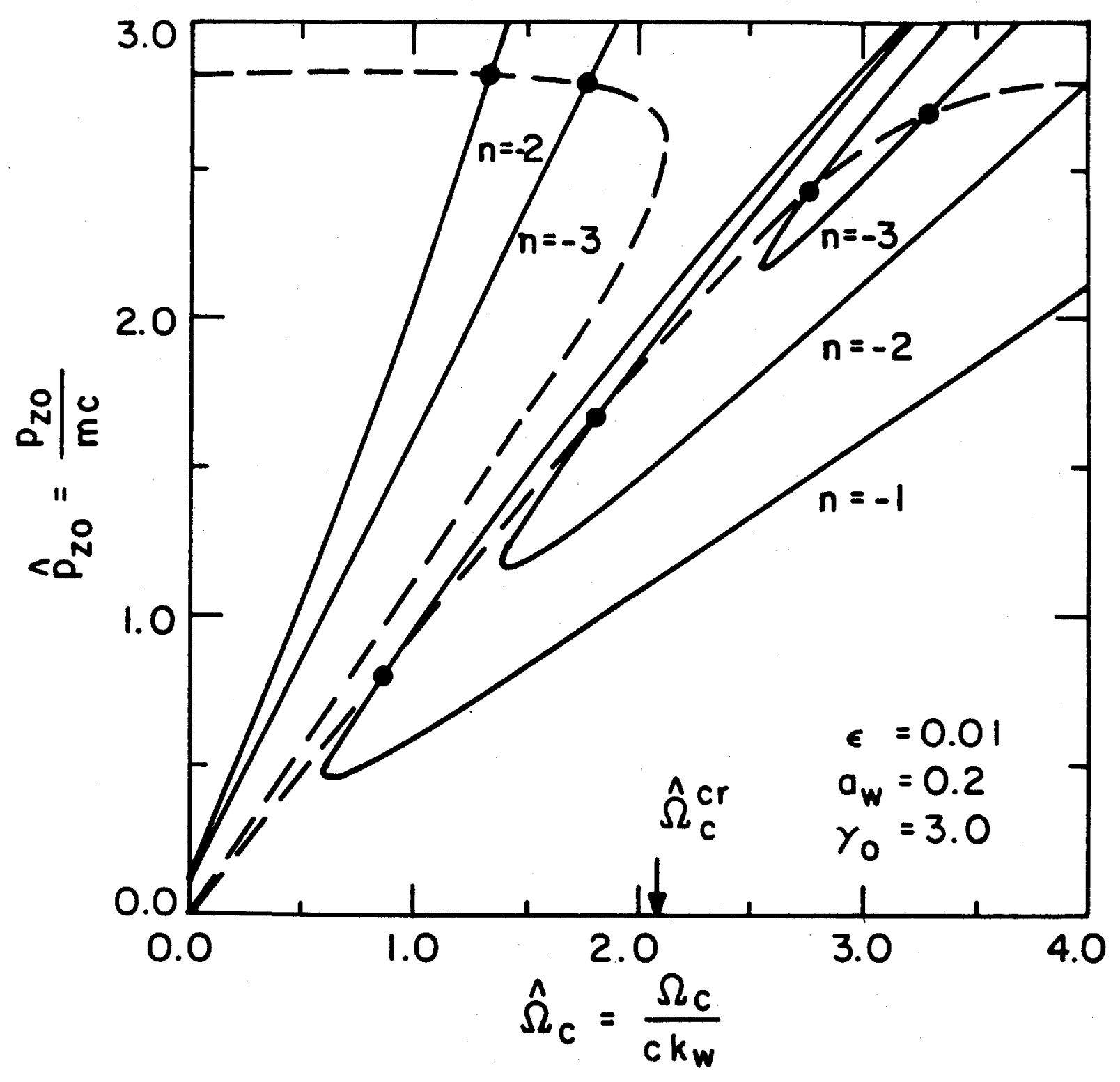

Figure 3 


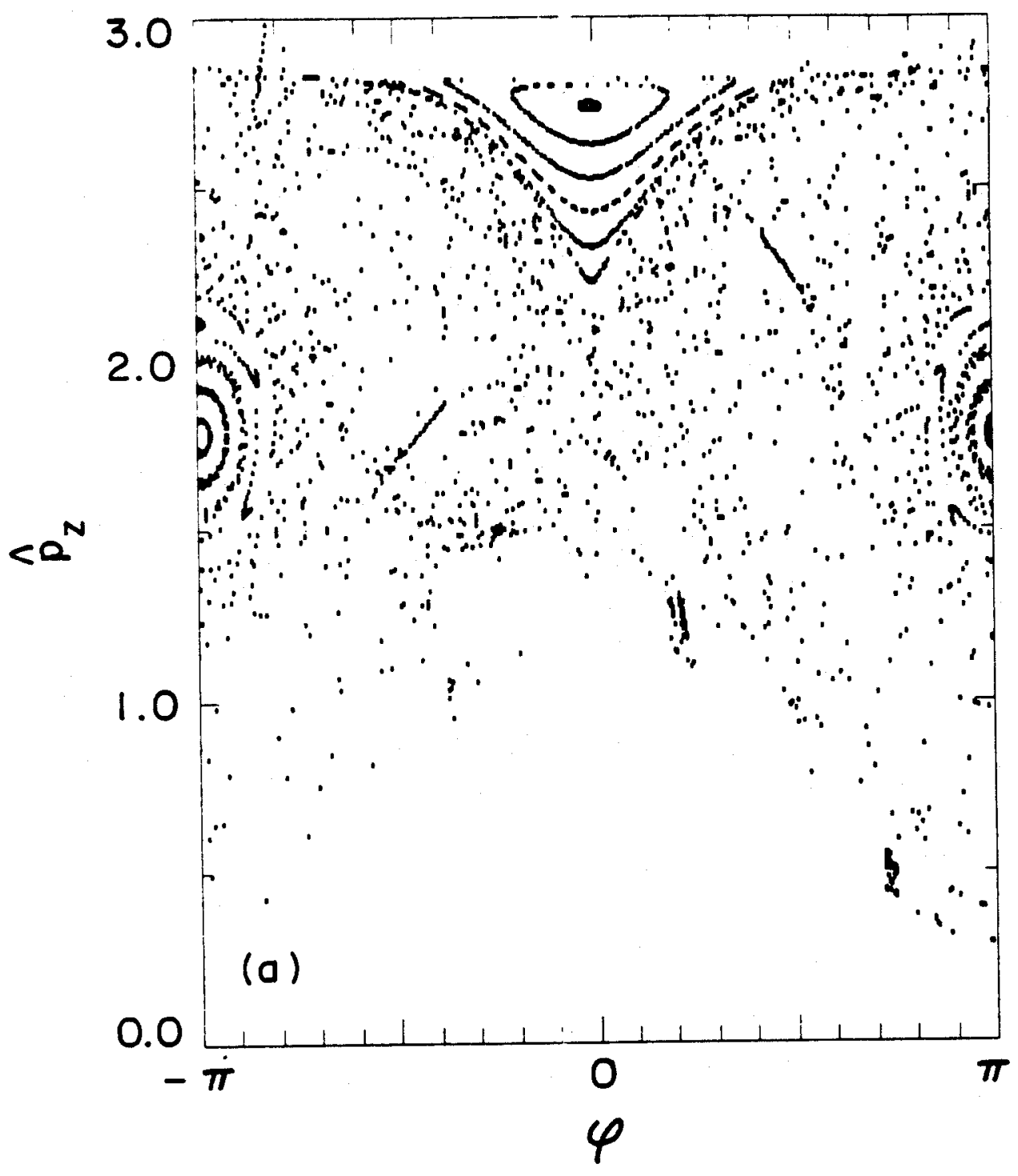

Figure $4 a$ 


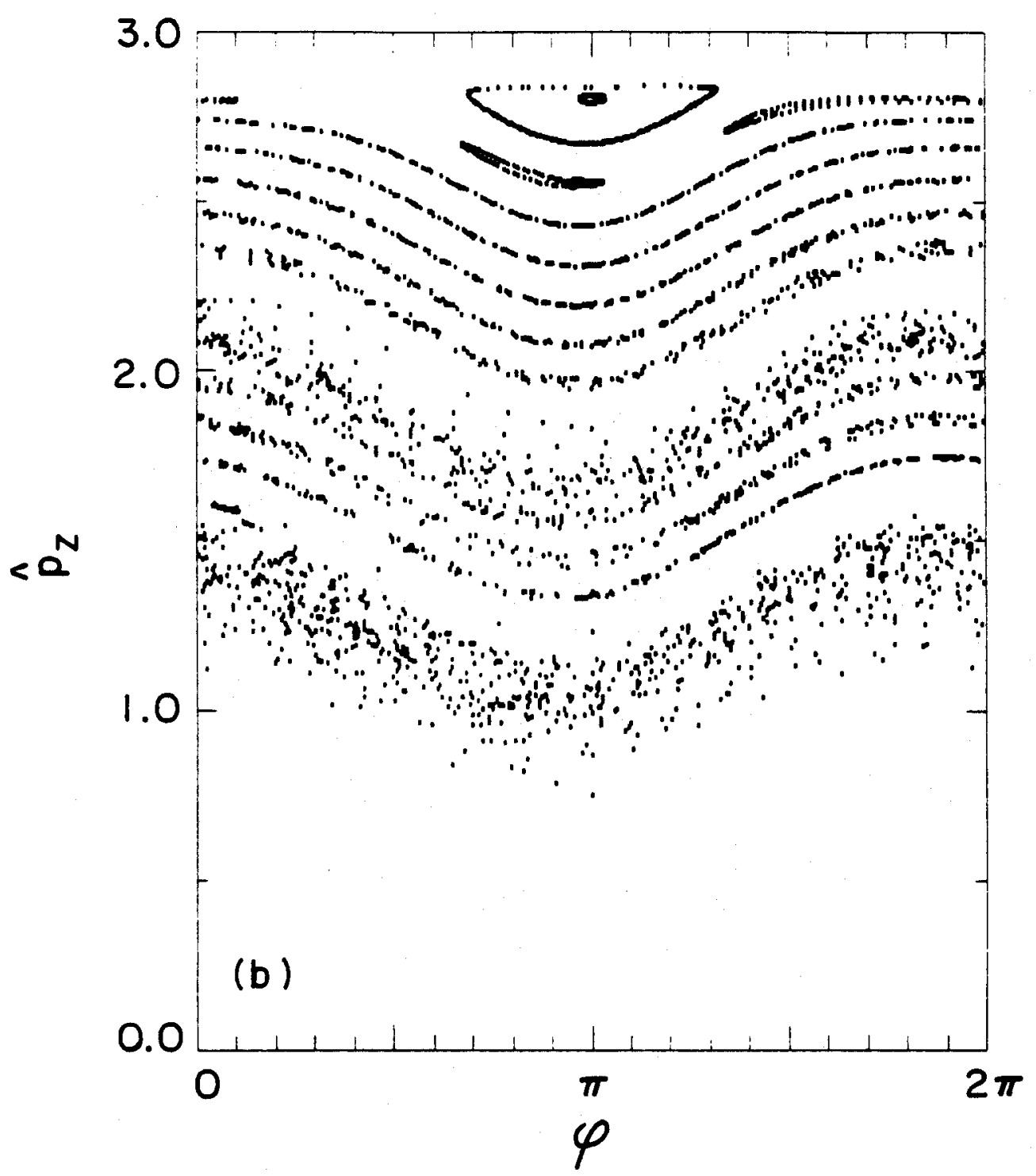

Figure 4b 


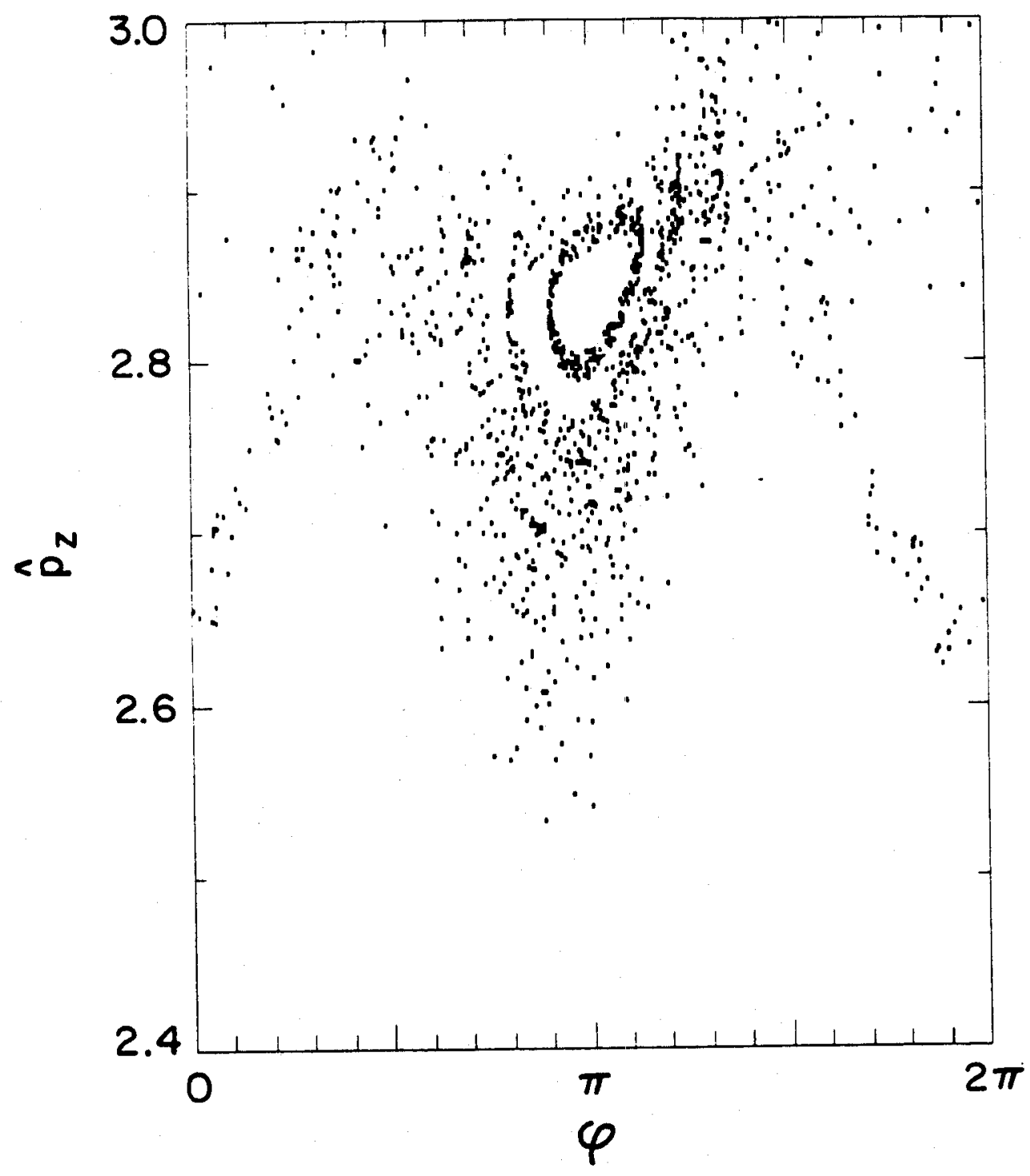

Figure 5 